Beach, A. S. (1952). J. gen. Microbiol. 6, 60-63.

\title{
An Agar Diffusion Method for the Assay of Nisin
}

\author{
By A. S. BEACH
}

Fisons Fine Chemical and Biological Laboratories, Loughborough, Leicestershire

SUMMARY: The diffusion rate of nisin through agar at $\mathrm{pH} \mathrm{7.0}$ is increased by the addition of a non-ionic surface active agent to the medium. A linear relationship exists between $\log$ units nisin/ml. and inhibition zone depth over the range 100 5000 units $/ \mathrm{ml}$. By using seeded agar in small glass tubes and delaying growth of the test organism for $24 \mathrm{hr}$., a convenient form of assay is available for dealing with large numbers of samples.

\section{EXPERIMENTAL}

Using the Oxford cylinder technique, no zones of inhibition were obtained when plates were incubated immediately after setting up, but when growth of the test organism was delayed by refrigeration for $24-48 \mathrm{hr}$. to allow time for the nisin to diffuse, some inhibition was apparent after subsequent incubation. Low concentrations of Tween 80 (Atlas Powder Co.) in the medium (Stansly \& Schlosser, 1947) considerably increased the diffusion rate of nisin in agar. Some compounds of the Crill series (non-ionic surface active agents manufactured by Croda Ltd., Goole, Yorks.) had an even greater effect (Fig. 1). Thus it became possible, by incorporating $0 \cdot 1 \%$ Crill 20 in the medium, to carry out agar diffusion assays provided that the plates were held in the refrigerator for $24 \mathrm{hr}$. after setting up and before incubation. An adaptation of the method of Mitchison \& Spicer (1949), using narrow glass tubes containing nutrient agar seeded with the test organism, was found to be much more convenient than the use of plates.

\section{Procedure}

The routine finally adopted was as follows.

Test organism. Streptococcus agalactiae was used.

Medium. Peptone (Oxoid), $10 \mathrm{~g}$; Lab Lemco, $5 \mathrm{~g}$; sodium phosphate $\left(\mathrm{Na}_{2} \mathrm{HPO}_{4} .12 \mathrm{H}_{2} \mathrm{O}\right), 20 \mathrm{~g}$.; sodium chloride, $20 \mathrm{~g}$.; dissolved in $500 \mathrm{ml}$. tap water, boiled at $\mathrm{pH} \mathrm{8.4}$ and filtered. To this was added an equal volume of $1 \%$ New Zealand agar in tap water, together with glucose, 5 g., and Crill 20, $0.5 \mathrm{~g}$. The reaction of the complete medium was adjusted, if necessary, to $\mathrm{pH} 8 \cdot \mathbf{0}$. This fell to $\mathrm{pH} \mathbf{7 \cdot 4}$ during sterilization.

Diluent. $0.02 \mathrm{~N}$-hydrochloric acid. This gave a standard response gradient equivalent to the best of those obtained with buffer solutions at various $\mathrm{pH}$ levels (Fig. 2).

Tubes. $100 \times 7 \mathrm{~mm}$. glass tubes (Franco British Electric Co.) fulfilled the requirements of having sufficient diameter to facilitate filling, cleaning and pipetting, of being robust enough to stand considerable handling, and of being economical in agar consumption. It has been reported (Hirsch, 1950) 
that nisin adheres to glass surfaces from which it is extremely difficult to remove. Attempts to produce this condition experimentally failed, and no nisin could be demonstrated in tubes which had been in use for the equivalent of fifty assays, during which time the tubes were washed only in plain water. Ordinary laboratory detergents were used for the routine cleaning of all glassware, but if not rinsed very thoroughly, residues of detergent caused distortion of inhibition zones.

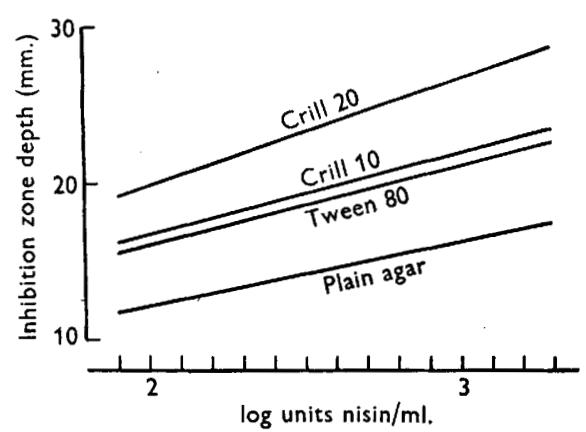

Fig. 1

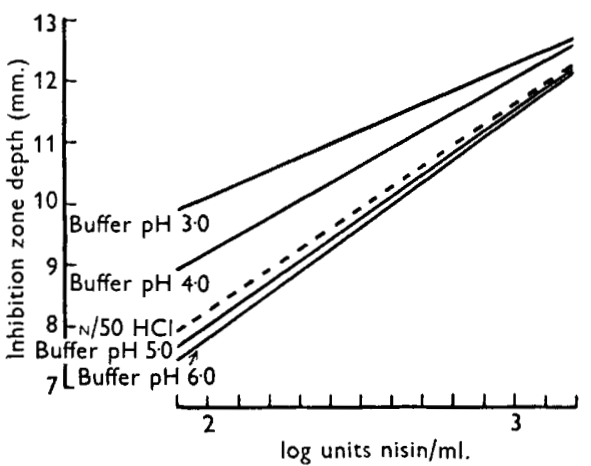

Fig. 2

Fig. 1. Effect of Tween 80 and Crill 20 on diffusion of nisin through agar. (Crill 10 is chemically identical with Tween 80.) Agar plates and porcelain cups were used in this experiment.

Fig. 2. Mean slopes of results from dilutions of nisin prepared in phosphate-citric acid buffers of different $\mathrm{pH}$ values and in $0.02 \mathrm{~N}$-hydrochloric acid.

Racks. Strips of $\frac{3}{8}$ in. Perspex approximately $12 \times 2 \frac{1}{2}$ in., drilled with four rows of eighteen holes and sealed on the under side with strips of $\frac{1}{8} \mathrm{in}$. Perspex.

Standard. Powder $118 \mathrm{~K}$ was assigned a value of 10 million units/g. based on the original definition of the nisin unit (Mattick \& Hirsch, 1947); it was stored in a desiccator at $5^{\circ}$ as a master standard. Other purified powders were used as subsidiary standards, fresh solutions containing 10,000 units $/ \mathrm{ml}$. were prepared each week in $0.02 \mathrm{~N}-\mathrm{HCl}$ and stored at $5^{\circ}$. The responses of a series of dilutions of nisin from 0 to 10,000 units $/ \mathrm{ml}$. were plotted. A marked bend in the slope was apparent (Fig. 3) so that it was decided to use two standard ranges, 0-100, and 125-2000 units $/ \mathrm{ml}$., according to the estimated potency of the samples to be tested.

\section{Method}

The tubes (unplugged) are sterilized and two-fifths filled with molten agar previously cooled to $43^{\circ}$ and seeded with $1 \%(\mathrm{v} / \mathrm{v})$ of a $6 \mathrm{hr}$. culture of Strep. agalactiae grown in glucose Lemco peptone broth. The tubes are stood upright until the agar has set, after which they may be stored in the refrigerator until required. When dealing with large numbers it is convenient to fill tubes on one day for use on the next.

Five serial dilutions of the standard are prepared ranging from 2000 to 125 units $/ \mathrm{ml}$.; for low potency samples the range is from 100-2 units $/ \mathrm{ml}$.; $\mathbf{0 . 1} \mathrm{ml}$. or more of each of the standard dilutions is added to three sets of four 
tubes, so that the final arrangement of the standard rack is three blocks of tubes each containing four replicates of the five dilutions.

Unknown solids are dissolved in $0.02 \mathrm{~N}-\mathrm{HCl}$, while brews and extraction liquors are adjusted to $c$. $\mathrm{pH} \mathrm{2.0} \mathrm{with} \mathrm{HCl}$, using thymol blue and a spotting tile. Up to five dilutions in powers of 2 are then prepared according to the degree of accuracy required, and so that the estimated potency of the dilutions falls within the standard range; $\mathbf{0 . 1} \mathbf{~ m l}$. or more of each dilution is then added to four seeded agar tubes.

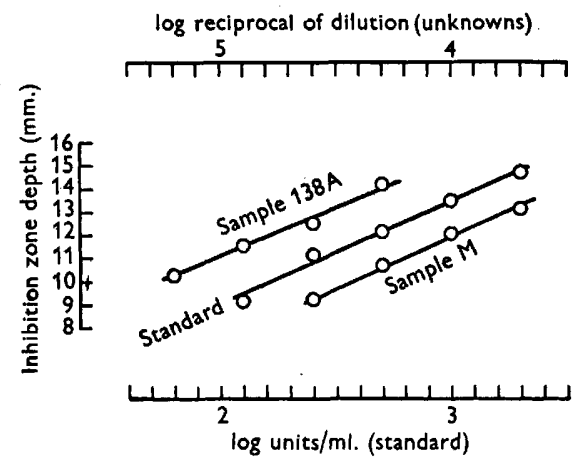

Fig. 3

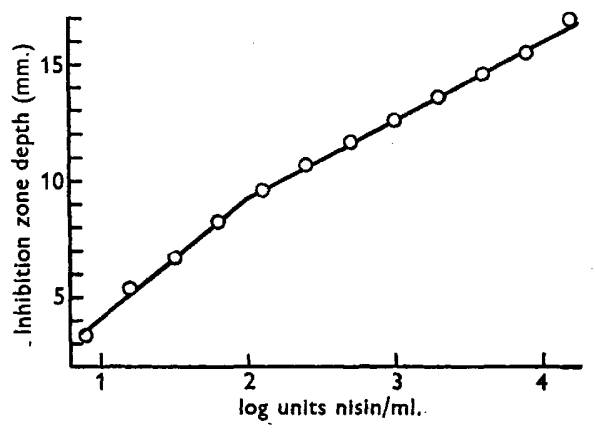

Fig. 4

Fig. 3. Mean slope of $10-10,000$ units nisin $/ \mathrm{ml}$.

Fig. 4. Typical assay results plotted for calculation.

Pasteur pipettes are used for dispensing solutions into the seeded agar tubes, and by rinsing the pipettes twice in $0.02 \mathrm{~N}-\mathrm{HCl}$ and once in the next sample to be handled, pipette consumption is decreased to a minimum. Using this technique, no carry over of nisin could be demonstrated experimentally, and it is thus possible to deal with large numbers of samples in a short space of time. Fresh standards are put up every hour when the time of setting up assays extends beyond this period. All the tubes are left in the refrigerator for $24 \mathrm{hr}$. to allow nisin to diffuse, and are then incubated at $37^{\circ}$ overnight.

Zone readings are usually made by direct vision with the aid of a millimetre scale marked on Perspex and with top illumination against a dark background. Other methods of reading using various means of magnification have been used, but for most purposes the advantage of speed outweighs that of slightly increased precision. Differences of $\mathbf{0 . 2 5} \mathrm{mm}$. can be detected without undue strain.

Averages of the readings of zone depth from each set of four tubes in the standard rack are plotted against log units nisin/ml., a straight line being drawn as the mean of these points. Readings from tubes containing unknown solutions are plotted in a similar manner on the same graph, but against the logarithm of the reciprocal of the dilution. The sum of log units $/ \mathrm{ml}$. from the standard slope, and log dilution from the slope of the unknown, at any suitable level, is the antilog of the potency of the sample (Fig. 4). Parallelism 
of the slopes is an indication of the validity of the assay; to eliminate error due to lack of parallelism, readings are taken at a level which intersects the middle of the slope of the unknown.

\section{DISCUSSION}

This form of assay has been used successfully for a considerable time, and except for the additional $\mathbf{2 4} \mathrm{hr}$. delay in obtaining results it has many advantages. Although the assay tubes are sterilized before being filled with seeded agar, they are not at any time plugged nor are any aseptic precautions taken in carrying out the assay. Pipettes, dilution tubes, etc., are not sterilized, and no trouble with contamination has been encountered over a long period. The volume of solution placed on the agar column in the assay tubes is immaterial so long as this is more than $0.1 \mathrm{ml}$.; with smaller volumes some absorption takes place. The volume of agar in the tubes is unimportant so long as it is sufficient for the greatest depth of inhibition zone expected, and as the ratio between surface area and available agar volume is always constant, slight differences in tube diameters do not introduce any error. Seeded agar tubes may be stored in the refrigerator for at least a week without deterioration and are thus available at any time. This is in contrast to the standard cupplate method where agar depth, cup diameter, etc., can affect the accuracy of the assay. Handling of tubes of this size is more convenient and they are far less bulky than Petri dishes or other types of agar plates in common use. The assay may be interpreted by the usual statistical procedures; thirty repetitions on one sample which was included daily along with routine samples gave a standard error of $5 \%(P=0.95)$.

I wish to thank Dr R. Falconer for encouragement and interest in this work, and to express appreciation to colleagues and staff for helpful criticisms and technical assistance. Publication is by permission of the Directors of Fisons Ltd.

\section{REFERENCES}

HIrsch, A. (1950). The assay of the antibiotic nisin. J. gen. Microbiol. 4, 70.

MatTick, A. T. R. \& Hirsch, A. (1947). Further observations on an inhibitory substance (nisin) from lactic streptococci. Lancet, ii, 5.

Mrtchison, D. A. \& Spicer, C. C. (1949). A method of estimating streptomycin in serum and other body fluids by diffusion through agar enclosed in glass tubes. J. gen. Microbiol. 3, 184.

Stansly, P. G. \& Schlosser, M. E. (1947). Studies on polymyxin: an agar diffusion method of assay. J. Bact. 54, 585 .

(Received 18 June 1951) 